Analele Universităţii de Vest, Timişoara

Seria Matematică - Informatică

LII, 2, (2014), 225- 230

\title{
On First Countability of a New Hyperspace Topology
}

Ritu Sen

\begin{abstract}
In this paper the notion of first countability of the hyperspace $(\theta(X), \tau)$ has been studied and being compared with that of $X$.
\end{abstract}

AMS Subject Classification (2000). 54B20

Keywords. $\theta$-open sets, $H$-sets, hemi $H$-closed space, locally $H$-space

\section{Introduction}

The study of hyperspace topology started in 1927 with Hausdorff [6] where he has topologized a collection of subsets of a topological space by defining a metric on the collection of all nonempty closed subsets of a bounded metric space $X$. Vietoris then introduced a new topology on the collection of all nonempty closed subsets of a topological space $(X, \tau)$, which is known as "Vietoris topology" or "Finite topology". After that, Michael in his paper [7] dealt with different types of subsets for the construction of topology. Subsequently, Fell introduced a compact, Hausdorff topology for the space of all closed subsets of a topological space. After that much of work has been done on hyperspace topology. In [5], the authors have introduced a new topology $\tau$ on the collection $\theta(X)$ of all nonempty $\theta$-closed subsets of a topological space $X$. 
In section 2, we recall some useful definitions and theorems. In section 3, we discuss the first countability of $(\theta(X), \tau)$. In the last theorem, we give the conditions under which the first countability of the topological space $X$ and $(\theta(X), \tau)$ are related.

\section{Preliminaries}

In this article we first discuss about the new hyperspace topology $\tau$ on the collection $\theta(X)$ of all nonempty $\theta$-closed subsets of a topological space $X$. For this we first recall some results discussed in [5]. Throughout the paper, $X$ will always mean a topological space. By a neighborhood of a point $x$ of a space $X$ we mean a subset $A$ of $X$ such that $x \in \operatorname{int}(A)$.

Definition 2.1. [9] A point $x \in X$ is said to be a $\theta$-contact point of a set $A \subseteq X$ if for every neighbourhood $U$ of $x$, we get $c l U \cap A \neq \varnothing$. The set of all $\theta$-contact points of a set $A$ is called the $\theta$-closure of $A$ and we denote this set by $\operatorname{cl}_{\theta} A$. $A$ set $A \subseteq X$ is called $\theta$-closed if $A=\operatorname{cl}_{\theta} A$. A set $A$ is called $\theta$-open if $X \backslash A$ is $\theta$-closed.

Note 2.1. The collection of all $\theta$-open sets in $X$ forms a topology $\tau_{\theta}$ on $X$ which is coarser than the original topology of $X$.

Notation 2.1. In this paper, $\theta(X)=\{A \subseteq X: A$ is nonempty $\theta$-closed $\}$.

Definition 2.2. [1] $A T_{2}$ space $X$ is called $H$-closed if any open cover of $X$ by means of open sets in $X$ has a finite proximate subcover i.e., a finite collection whose union is dense in $X . A$ set $A \subseteq X$ is called an $H$-set if any open cover $\left\{U_{\alpha}: \alpha \in \Lambda\right\}$ of $A$ by open sets of $X$ has a finite subfamily $\left\{U_{\alpha_{i}}: i=1,2, \ldots, n\right\}$ such that $A \subseteq \cup_{i=1}^{n} c l U_{\alpha_{i}}$.

Theorem 2.2. [2] In an $H$-closed Urysohn space, every $H$-set is $\theta$-closed and every $\theta$-closed set is an $H$-set.

Note 2.3. If we look at the definition of $H$-sets, we see that the cover must consist of open sets of the whole space. If the covers be formed by open sets of the subspace, then the same condition gives rise to an $H$-closed subspace, which can be proved easily to be an $H$-set. But there are $H$-sets which are not $H$-closed subspaces. Thus the class of $H$-sets contains the class of $H$-closed subspaces of a space, but does not coincide with it in general.

Notation 2.2. For a space $X, H(X)$ will denote the collection of all nonempty $H$-sets of $X$. 
Definition 2.3. [5] On $\theta(X)$ we define a topology as follows :

For each $W \subseteq X$, let $W^{+}=\{A \in \theta(X): A \subseteq W\}$ and $W^{-}=\{A \in \theta(X)$ : $A \cap W \neq \varnothing\}$. Consider $S_{\theta}=\left\{W^{-}: W\right.$ is open in $\left.X\right\} \cup\left\{W^{+}: W\right.$ is $\theta$-open in $X$ and $X \backslash W$ is an $H$-set $\}$. Then $S_{\theta}$ forms a subbase for some topology on $\theta(X)$ which we denote by $\tau$.

Note 2.4. Any basic open set in the above defined topology $\tau$ is of the form $V_{1}^{-} \cap \ldots \cap V_{n}^{-} \cap V_{0}^{+}$where $V_{i} \subseteq V_{0}$ for $1 \leq i \leq n$, and $V_{1}, V_{2}, \ldots V_{n}$ are open sets, $V_{0}$ is a $\theta$-open set with $X \backslash V_{0}$ an $H$-set.

Notation 2.3. If $\alpha=\left\{V_{1}, \ldots, V_{n}\right\}$ is a finite family of open subsets of a space $X$ then we set $\alpha^{-}=\cap\left\{V_{i}^{-}: i=1, \ldots, n\right\}$.

Definition 2.4. A $T_{2}$ space is called a locally $H$-space if every point of it has a neighborhood which is an $H$-set.

Definition 2.5. A family $\mathcal{H} \subseteq H(X)$ is said to be cofinal in $H(X)$ if for any $H \in H(X)$, there exists $H^{\prime} \in \mathcal{H}$ such that $H \subseteq H^{\prime}$.

Definition 2.6. A topological space $X$ is called hemi $H$-closed if there is a countable subfamily of $H(X)$ which is cofinal in $H(X)$.

Theorem 2.5. [4] $X$ is $T_{2}$ if and only if $\{x\}$ is $\theta$-closed for each $x \in X$.

\section{On first countability of $X$ and $(\theta(X), \tau)$}

In this article we discuss the first countability for the hyperspace $(\theta(X), \tau)$ and endeavour has been made to study, how the first countability of $(\theta(X), \tau)$ is related to the first countability of the topological space $X$.

Definition 3.1. The tightness of a space $X$ is said to be countable, if for each $C \subseteq X$ and each $x \in X$ with $x \in \operatorname{cl}(C)$, there exists a countable subset $C_{0}$ of $C$ such that $x \in \operatorname{cl}\left(C_{0}\right)$.

Proposition 3.1. Let $X$ be a $T_{2}$ space. If the tightness of $(\theta(X), \tau)$ is countable, then any $A \in \theta(X)$ is separable.

Proof. Let $A \in \theta(X)$. Let

$$
\mathcal{F}=\{E: E \text { is finite subset of } A\} .
$$


We first show that $A \in c l \mathcal{F}$. Let $U_{1}^{-} \cap U_{2}^{-} \cap \ldots \cap U_{n}^{-} \cap U_{0}^{+}$be a basic open neighbourhood of $A$, where $U_{1}, U_{2}, \ldots U_{n}$ are open in $X$ and $U_{0}$ is $\theta$-open in $X$ with $X \backslash U_{0}$ an $H$-set. Then $A \cap U_{i} \neq \varnothing$ for each $i=1,2, \ldots, n$. Choose $a_{i} \in A \cap U_{i}$ for $i=1,2, \ldots, n$ and let $F=\left\{a_{1}, a_{2}, \ldots, a_{n}\right\}$. Then $F \in \mathcal{F}$. Also $A \subseteq U_{0}$ i.e., $A \cap\left(X \backslash U_{0}\right)=\varnothing$ which implies that $F \cap\left(X \backslash U_{0}\right)=\varnothing$. Hence $F \in U_{1}^{-} \cap U_{2}^{-} \cap \ldots \cap U_{n}^{-} \cap U_{0}^{+} \cap \mathcal{F}$. Since the tightness of $(\theta(X), \tau)$ is countable, there exists a countable subfamily $\mathcal{F}^{\prime} \subseteq \mathcal{F}$ such that $A \in \operatorname{cl} \mathcal{F}^{\prime}$. Set $G=\cup \mathcal{F}^{\prime}$. Then $A \subseteq c l G$. Indeed, let $a \in A$ and $V$ be an open neighbourhood of $a$. Then $A \in V^{-}$. Hence $V^{-} \cap \mathcal{F}^{\prime} \neq \emptyset$. Thus there exists $F \in \mathcal{F}^{\prime}$ such that $F \cap V \neq \emptyset$. Then $G \cap V \neq \emptyset$. Therefore $A \subseteq c l G$.

Proposition 3.2. Let $X$ be an $H$-closed Urysohn space. If $(\theta(X), \tau)$ is first countable, then every proper $\theta$-open subset of $X$ is hemi $H$-closed.

Proof. Let $U$ be a proper $\theta$-open subset of $X$. Let $V=X \backslash U$. Since $(\theta(X), \tau)$ is first countable, there is a local base $\mathcal{F}=\left\{\alpha_{i}^{-} \cap U_{i}^{+}: i \in N\right\}$ at $V \in \theta(X)$, where each $\alpha_{i}$ is a finite family of non-empty open subsets of $X$ and each $U_{i}$ is a $\theta$-open subset of $X$ with $X \backslash U_{i}$ an $H$-set for $i \in N$. We now show that $\mathcal{U}=\left\{X \backslash U_{i}: i \in N\right\}$ is cofinal in $H(U)$ which proves that $U$ is hemi $H$-closed. Let $P \in H(U)$. Then $P \cap V=\varnothing$ i.e., $V \in(X \backslash P)^{+}$, where $X \backslash P$ is $\theta$-open as $P$ being an $H$-set in $U$, it is so in $X$ and as $X$ is $H$-closed and Urysohn, $P$ is $\theta$-closed. Then there exists $i \in N$ such that $V \in \alpha_{i}^{-} \cap U_{i}^{+} \subseteq(X \backslash P)^{+}$. If $P \backslash\left(X \backslash U_{i}\right) \neq \varnothing$, then for $x \in P \backslash\left(X \backslash U_{i}\right),\{x\} \cap\left(X \backslash U_{i}\right)=\varnothing$ and so $\{x\} \cup V \in \alpha_{i}^{-} \cap U_{i}^{+}$, but $\{x\} \cup V \notin(X \backslash P)^{+}$which is a contradiction. Hence $P \subseteq\left(X \backslash U_{i}\right)$ and hence the proposition is proved.

Theorem 3.3. Let $X$ be a first countable, $H$-closed, Urysohn space. Then $(\theta(X), \tau)$ is first countable if and only if each $A \in \theta(X)$ is separable and every proper $\theta$-open subset of $X$ is hemi $H$-closed.

Proof. If $(\theta(X), \tau)$ is first countable, then by Proposition 3.1, and Proposition 3.2 , every $\theta$-closed subset of $X$ is separable and every proper $\theta$-open subset of $X$ is hemi $H$-closed.

Conversely, let every $\theta$-closed subset of $X$ be separable and every proper $\theta$ open subset of $X$ be hemi $H$-closed. Let $A \in \theta(X)$. Then by hypothesis, $A$ is separable and hence there exists a countable subset $G$ of $A$ such that $A=c l G$. Since $X$ is first countable, there is a countable local base $\mathcal{V}_{g}$ at $g$ for every $g \in G$. Define $\mathcal{V}=\cup\left\{\mathcal{V}_{g}: g \in G\right\}$ and let $\mathcal{U}$ be the set of all finite subsets of $\mathcal{V}$. Since $X \backslash A$ is hemi $H$-closed, there exists a countable family $\mathcal{H} \subseteq H(X \backslash A)$ which is cofinal in $H(X \backslash A)$. Let $\mathcal{W}=\left\{U^{-} \cap K^{+}: U \in \mathcal{U}, X \backslash K \in \mathcal{H}\right\}$. Then $\mathcal{W}$ is countable. We now show that $\mathcal{W}$ is a local base at $A \in \theta(X)$. Let $U_{1}^{-} \cap U_{2}^{-} \cap \ldots \cap U_{n}^{-} \cap U_{0}^{+}$be a basic neighbourhood of $A$, where each $U_{i}$, for 
$i=1,2, \ldots, n$ is open in $X$ and $U_{0}$ is a $\theta$-open subset of $X$ with $X \backslash U_{0}$ an $H$ set. Then $A \cap U_{i} \neq \varnothing$ for $i=1,2, \ldots, n$ and $A \subseteq U_{0}$. Now there exists $H \in \mathcal{H}$ such that $X \backslash U_{0} \subseteq H$ and $\left\{V_{1}, V_{2}, \ldots, V_{n}\right\} \in \mathcal{U}$ such that $x_{i} \in V_{i} \subseteq U_{i}$ for $x_{i} \in U_{i} \cap G, 1 \leq i \leq n$. Thus $A \in V_{1}^{-} \cap V_{2}^{-} \cap \ldots \cap V_{n}^{-} \cap(X \backslash H)^{+} \subseteq \cap_{i=1}^{n} U_{i}^{-} \cap U_{0}^{+}$ so that $(\theta(X), \tau)$ is first countable.

Proposition 3.4. Let $X$ be a first countable $T_{2}$ space. If each proper $\theta$-open subset of $X$ is hemi $H$-closed, then $X$ is a locally $H$-space.

Proof. Let $x \in X$. If $X=\{x\}$, we are done. Now let there exists some point $p \in X \backslash\{x\}$. Let $U=X \backslash\{p\}$. Then $U$ is a proper $\theta$-open subset of $X$ and hence by hypothesis, there exists an increasing sequence $\left\{H_{n}: n \in N\right\}$ of $H$-sets of $U$ such that every $H$-set of $U$ is contained in some $H_{n}$. Since $X$ is first countable, there is a decreasing sequence $\mathcal{V}=\left\{V_{n}: n \in N\right\}$ which is a neighbourhood base at $x$ and $V_{1} \subseteq U$. We now show that there exists $n \in N$ such that $V_{n} \subseteq H_{n}$. If not, then for every $n \in N$, we can choose $x_{n} \in V_{n} \backslash H_{n}$, so that the sequence $\left\{x_{n}: n \in N\right\}$ converges to $x$. Hence there exists $k \in N$ such that $\left\{x_{n}: n \in N\right\} \cup\{x\} \subseteq H_{k} \Rightarrow x_{k} \in H_{k}$, a contradiction. Hence the proposition is proved.

Theorem 3.5. Let $X$ be a $T_{2}$ topological space. If $(\theta(X), \tau)$ is first countable, then so is $X$.

Proof. Let $x \in X$. Since $(\theta(X), \tau)$ is first countable, there exists a local base $\mathcal{W}=\left\{\alpha_{i}^{-} \cap K_{i}^{+}: i \in N\right\}$ at $\{x\}$, where $\alpha_{i}$ is a finite family of open subsets of $X, K_{i}$ is $\theta$-open in $X$ and $X \backslash K_{i}$ is an $H$-set in $X$. Define $\mathcal{V}=\left\{K_{i} \cap\left(\cap \alpha_{i}\right): i \in N\right\}$. We now show that $\mathcal{V}$ is a local base at $x \in X$. Now for each $V \in \mathcal{V}, x \in V$ and $V$ is open in $X$. Let $U$ be an open neighbourhood of $x \in X$. Then $\{x\} \in U^{-}$. Then there exists $i \in N$ such that $\{x\} \in \alpha_{i}^{-} \cap K_{i}^{+} \subseteq U^{-}$. Clearly $x \in K_{i} \cap\left(\cap \alpha_{i}\right) \subseteq U$. Hence $X$ is first countable.

\section{Acknowledgement}

The author is thankful to the referee for his valuable comments to improve the quality of the paper. 


\section{References}

[1] Alexandröff P. S. and Urysohn P., Mémoire sur les espaces topologiques compacts, Verhandelingen der Koninklijke Akademie ven Wetenschappen te Amsterdam, 14, (1929), 1-96

[2] Dickman R. F. Jr. and Porter J. R., $\theta$-perfect and $\theta$-absolutely closed functions, Illinois J. of Math., 21, 42, (1977), 42-60

[3] Fell J. M. G., A Hausdorff topology for the closed subsets of a locally compact non-Hausdorff space, Proc. Amer. Math. Soc., 13, (1962), 472-476

[4] Ganguly S. and Jana S., A note on partially oredred topological spaces and a special type of lower semicontinuous function, Carpathian J. Math., 21, (2005), 61-67

[5] Ganguly S., Jana S., and Sen R., A new hyperspace topology and the study of the function space $\theta^{*}-L C(X, Y)$, Matematicki Vesnik, 2009, 181-193

[6] Hausdörff F., Mengenlehre, 3d ed., Springer, Berlin, 1927

[7] Michael E., Topologies on spaces of subsets, Trans. Amer. Math. Soc., 71, (1951), $152-182$

[8] Sen R., Local connectedness in hyperspace topology, Anal. Univ. Oradia Fasc. Mat., Tom XX 1, (2013), 117-123

[9] Veličko N. V., H-closed topological spaces, Amer. Math. Transl., 78, (1968), 103-118

\section{Ritu Sen}

Department of Mathematics

S. A. Jaipuria College

10, Raja Naba Krishna Street

Kolkata 700005

INDIA

E-mail: ritu_sen29@yahoo.co.in

Received: 30.12.2013

Revised: 25.09.2014

Accepted: 3.11.2014 